\title{
Development of a Reference Database for Particle-Induced Gamma-ray Emission spectroscopy
}

\author{
P. Dimitriou ${ }^{a, *}$, H.-W. Becker ${ }^{b}$, I. Bogdanović-Radović ${ }^{c}$, M. Chiari ${ }^{d}$, A. Goncharov ${ }^{\text {e }}$, A.P. Jesus ${ }^{f}$, \\ O. Kakuee ${ }^{g}$, A.Z. Kiss ${ }^{\text {h}}$, A. Lagoyannis ${ }^{i}$, J. Räisänen ${ }^{j}$, D. Strivay ${ }^{k}$, A. Zucchiatti $^{1}$ \\ a International Atomic Energy Agency, Wagramerstrasse 5, A-1400 Vienna, Austria \\ ${ }^{\mathrm{b}}$ Ruhr Universität Bochum, Gebäude NT05/130, Postfach 102148, Bochum 44721, Germany \\ ${ }^{\mathrm{C}}$ Department of Experimental Physics, Institute Rudjer Boskovic, Bijenicka Cesta 54, 10000 Zagreb, Croatia \\ ${ }^{\mathrm{d}}$ Istituto Nazionale di Fisica Nucleare, Via Sansone 1, Sesto Fiorentino, 50019 Firenze, Italy \\ ${ }^{\mathrm{e}}$ Kharkov Institute of Physics and Technology, National Science Center, Akademicheskaya Str.1, Kharkov 61108, Ukraine \\ ${ }^{\mathrm{f}}$ Departamento de Física, Faculdade de Ciências e Tecnologia da Universidade Nova de Lisboa, Portugal \\ ${ }^{\mathrm{g}}$ Nuclear Science and Technology Research Institute, End of North Karegar Ave., PO Box 14395-836, Tehran, Iran \\ ${ }^{\mathrm{h}}$ Institute of Nuclear Research (ATOMKI), Bem ter 18/c, PO Box 51, 4001 Debrecen, Hungary \\ ${ }^{i}$ National Center of Scientific Research "Demokritos", Agia Paraskevi, P.O. Box 60228, 15310 Athens, Greece \\ ${ }^{\mathrm{j}}$ Division of Materials Physics, Department of Physics, University of Helsinki, PO Box 43, 00014 University of Helsinki, Finland \\ ${ }^{\mathrm{k}}$ Institut de Physique Nucleaire, Atomique et de Spectroscopie, Universite de Liège, Sart Tilman, B15 4000 Liège, Belgium \\ ${ }^{1}$ Centro de Micro Análisis de Materiales, Universidad Autónoma de Madrid, Faraday 3, Madrid 28049, Spain
}

\section{A R T I C L E I N F O}

\section{Article history:}

Received 10 July 2015

Received in revised form 14 September 2015

Accepted 16 September 2015

Available online $\mathrm{xxxx}$

\section{Keywords:}

Ion Beam Analysis

Differential cross-section

Measurement

Evaluation

\begin{abstract}
A B S T R A C T
Particle-Induced Gamma-ray Emission (PIGE) is a powerful analytical technique that exploits the interactions of rapid charged particles with nuclei located near a sample surface to determine the composition and structure of the surface regions of solids by measurement of characteristic prompt $\gamma$ rays. The potential for depth profiling of this technique has long been recognized, however, the implementation has been limited owing to insufficient knowledge of the physical data and lack of suitable user-friendly computer codes for the applications. Although a considerable body of published data exists in the nuclear physics literature for nuclear reaction cross sections with $\gamma$ rays in the exit channel, there is no up-to-date, comprehensive compilation specifically dedicated to IBA applications. A number of PIGE cross-section data had already been uploaded to the Ion Beam Analysis Nuclear Data Library (IBANDL) (http://www-nds. iaea.org/ibandl) by members of the IBA community by 2011, however a preliminary survey of this body of unevaluated experimental data has revealed numerous discrepancies beyond the uncertainty limits reported by the authors. Using the resources and coordination provided by the IAEA, a concerted effort to improve the situation was made within the Coordinated Research Project on the Development of a Reference Database for PIGE spectroscopy, from 2011 to 2015. The aim of the CRP was to create a data library for Ion Beam Analysis that contains reliable and usable data on charged particle $\gamma$-ray emission cross sections that would be made freely available to the user community. As the CRP has reached its completion, we shall present its main achievements, including the results of nuclear cross-section evaluations and the development of a computer code that will become available to the public allowing for the implementation of a standardless PIGE technique.
\end{abstract}

(c) 2015 Elsevier B.V. All rights reserved.

\section{Introduction}

Among the various accelerator-based Ion Beam Analysis techniques used for material characterization, Particle-Induced Gamma-ray Emission spectroscopy (PIGE) is one of the most

\footnotetext{
* Corresponding author.

E-mail address: P.Dimitriou@iaea.org (P. Dimitriou).
}

powerful ones. PIGE uses the interactions of rapid (several MeV) charged particles with matter to determine the composition and the depth profile of constituting elements near the surface (from 0 to $500 \mu \mathrm{m}$ ) by measuring the energy spectra of prompt $\gamma$ rays. This technique has been used since the early 1960's in different fields of applications ranging from analysis of fission rector materials to biomedicine, environment,cultural heritage, and more recently, fusion reactor materials. Its potential for depth profiling 

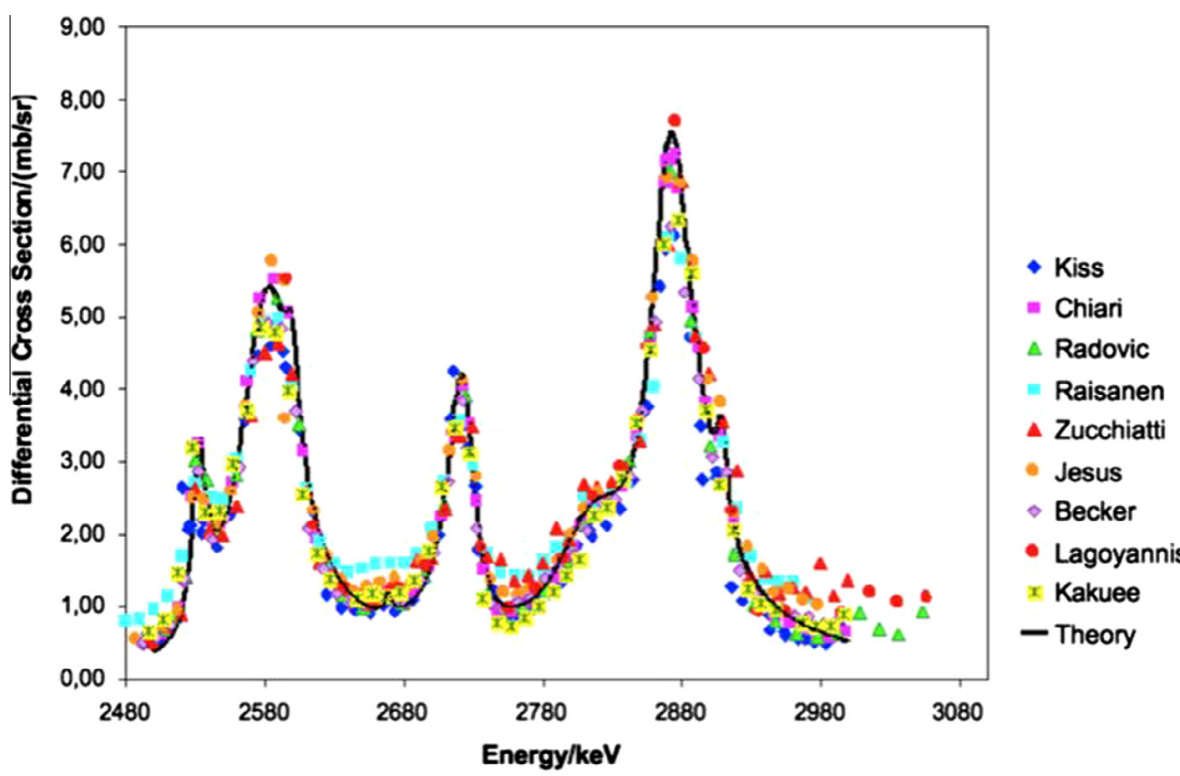

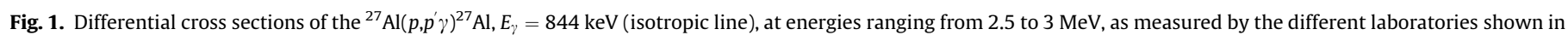
the legend. The solid line corresponds to the evaluated cross section [16].

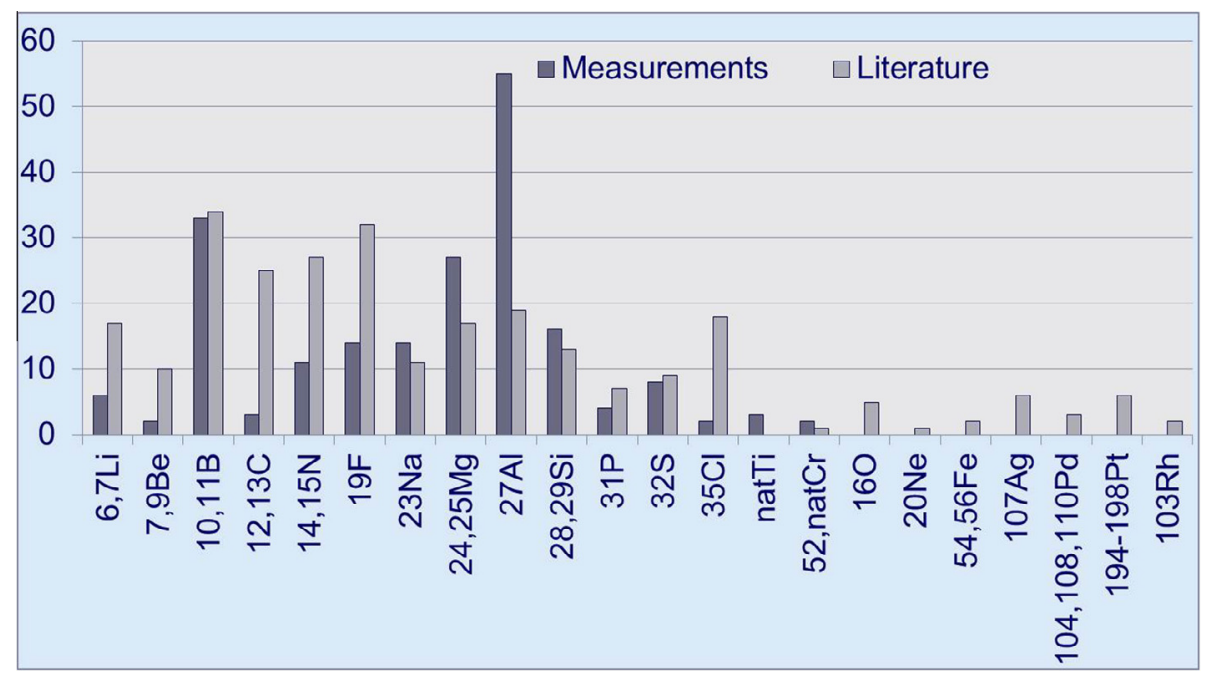

Fig. 2. Number of PIGE datasets available on IBANDL during the course of the PIGE CRP.

with better resolution than the other IBA techniques was recognized early on.

As with all IBA methods including backscattering spectroscopy and nuclear reaction analysis, PIGE relies strongly on differential cross-section data, the lack of which has significantly hindered progress in the implementation of IBA techniques in general. In an attempt to address the IBA data needs, the Nuclear Data Section (NDS) of the International Atomic Energy Agency (IAEA) held a Coordinated Research Project (CRP) on the Development of a Reference Database for Ion Beam Analysis [1] from 2005 to 2010. The CRP produced a comprehensive and reliable database of crosssection data relevant for elastic backscattering (EBS) and nuclear reaction analysis (NRA) techniques primarily, including evaluated cross sections. The experimental cross-section data were made available on the Ion Beam Analysis Nuclear Data Library (IBANDL) [2] while the evaluated cross sections are available through the online calculator SigmaCalc [3]. While this first CRP catered for the data needs of the EBS and NRA techniques, the situation for
PIGE was far from satisfactory. Although there existed a considerable body of published data in the nuclear physics literature, there was no up-to-date and comprehensive compilation dedicated to IBA purposes. Although a number of PIGE cross-section data had already been uploaded to IBANDL by members of the IBA community, a preliminary survey of this data revealed numerous discrepancies showing that there was an urgent need for compilation, assessment and evaluation of the PIGE cross-sections.

In response to the request of the IBA community, NDS organized a consultant's meeting to assess the data needs for PIGE and propose actions for improving the quality and availability of nuclear cross-section data for PIGE. The meeting was held in 2010 [4] and recommended a CRP with the goal to develop a Reference Database for PIGE data that would be freely available to the community of practice. The IAEA CRP ran from 2011 to 2015 with the participation of ten laboratories from ten different countries with active IBA research and applications programmes. Three Research Coordination Meetings (RCM) were held [5-7] during the course 
of the CRP to determine, monitor and revise the work plan which consisted of the following main activities:

- Compilation of existing data.

- New measurements.

- Assessment of existing and new data.

- Study of the feasibility of evaluating PIGE data.

- Development of a PIGE analysis code for bulk analysis and depth profiling.

These activities will be discussed in more detail in the following sections.

\section{Experimental data}

\subsection{New measurements}

New measurements of $\gamma$-production cross sections for thin targets constitute a major part of the activities of this CRP. The objective was to measure cross sections relevant to the analysis of elements of importance for the various applications such as sodium, aluminum, boron, fluorine, magnesium, sulfur, beryllium, silicon, nitrogen, oxygen and chromium. An initial survey of the literature revealed discrepancies among different authors, lack of data for some important nuclear reactions, as well as insufficient coverage of energy ranges needed for PIGE applications [4,5].

Ten different laboratories with different facilities covering a wide range of angles and energies participated in the measurement campaign. To arrive at a consistent set of absolute cross-section measurements that would allow for a meaningful comparison and assessment, a common methodology was adopted to treat the following experimental aspects [5]: (i) energy calibration of accelerators, (ii) target preparation, (iii) detector efficiency, (iv) cross-section normalization, and (v) assignment of uncertainties.

The majority of the new cross-section data have already been uploaded in IBANDL. Details of some of the measurements can be found in Refs. [8-15].

\subsection{Coordinated measurement}

A coordinated exercise was carried out in an effort to identify possible sources of discrepancies and systematic errors among the laboratories participating in the CRP. All ten groups measured the cross section of the reaction ${ }^{27} \mathrm{Al}\left(p, p^{\prime} \gamma\right){ }^{27} \mathrm{Al}, E_{\gamma}=844 \mathrm{keV}$ (isotropic line), at energies ranging from 2.5 to $3 \mathrm{MeV}$ with an energy step of $10 \mathrm{keV}$, for a thin target. A similar exercise was performed for a thick-target measurement: participants measured the yield of the reaction ${ }^{27} \mathrm{Al}(p, \gamma)^{28} \mathrm{Si}$ using the $E_{\gamma}=1.779 \mathrm{MeV} \gamma$ line, at energies ranging from 0.95 to $1.1 \mathrm{MeV}$ with an energy step small enough to reproduce the ${ }^{27} \mathrm{Al}(p, \gamma)^{28} \mathrm{Si}$ resonance at $991 \mathrm{keV}$ proton energy. The cross sections measured at the different facilities as well as details of the different experimental procedures outlined above, including the uncertainty budgets, were collected and critically assessed. Some results are shown in Fig. 1. A careful comparison of the resulting data for the thin-target measurements reveals an inconsistency in the energies of the narrow resonances that were used for energy calibration purposes, especially at energies above $2 \mathrm{MeV}$. As far as the cross-section values are concerned, the reported data agree within the quoted uncertainties (7-10\%), with one or two exceptions which need to be revisited.

The conclusions drawn from this coordinated measurement have been helpful in analyzing and assessing the rest of the reactions that have been measured for this CRP. In particular the realization that all the steps of the common methodology outlined above need to be treated with special care and well documented so that possible discrepancies can be easily traced back to their sources. Overall, one can safely say that provided the commonly accepted methodology is implemented with care and rigor, absolute PIGE measurements can be performed with an accuracy of up to $10 \%$. A detailed article on this coordinated measurement is in preparation.

\subsection{Literature}

Most of the existing data were already compiled in EXFOR [17] and were thus converted to R33 format through an automatized procedure that consists of a simple press of a button. Data published in graphical format were digitized by NDS staff using established software that are regularly used for EXFOR compilations. So far 265 data sets for thin- and thick-target measurements found in the existing literature have been uploaded into IBANDL.The search for existing data was extended to the databases and compilations used by the nuclear astrophysics community (NACRE [18]) since there is considerable overlap between the data needs for IBA applications and stellar nucleosynthesis studies.

\section{Evaluations}

Data evaluation consists of several well established and generally accepted steps such as data compilation, critical assessment of existing data, parameterization in the framework of a suitable nuclear model and statistical analysis of the inconsistencies between model calculations and experimental data. To meet the needs of the IBA techniques, a certain number of charged-particle cross sections, mainly for elastic scattering and transfer reactions, have been evaluated and the SigmaCalc software was developed for IBA scientists to perform calculations of differential cross sections at any angle. Similar evaluation efforts were applied to PIGE cross-section data within the CRP, and the SigmaCalc software was extended to provide evaluated cross sections for the following reactions [16,19]: ${ }^{27} \mathrm{Al}\left(p, p^{\prime} \gamma_{1-0}\right){ }^{27} \mathrm{Al},{ }^{23} \mathrm{Na}\left(p, p^{\prime} \gamma_{1-0}\right){ }^{23} \mathrm{Na}$, and ${ }^{53} \mathrm{Cr}$ $\left(p, \gamma_{1-0}\right)^{54} \mathrm{Mn}$. The results of the evaluation for ${ }^{27} \mathrm{Al}\left(p, p^{\prime} \gamma\right)^{27} \mathrm{Al}$ are shown in Fig. 1.

\section{PIGE analysis code}

One of the advantages of having a database with reliable and precise PIGE differential cross-section data readily available to the community of practice is that it would allow for the technique to be implemented without the need of a 'standard' to compare or normalize to. The aim of 'standardless' PIGE could only be achieved however, if besides the differential cross-section data, the community was also provided with a reliable computer code to calculate the material composition in the case of bulk analysis and the layer composition in the case of depth analysis using a given set of crosssection data and stopping power data. To this end, the ERYA code, which was developed in CFNUL, Universidade de Lisboa [20] for bulk analysis of sample materials using PIGE cross sections, was distributed among the CRP participants to enable them to test the PIGE Reference Database in specific cases of bulk sample analysis. The code was further developed to include depth analysis capabilities [21].

\section{Conclusions}

With the completion of the IAEA CRP on Development of a Reference Database for Particle-Induced Gamma-ray Emission spectroscopy, IBANDL includes a comprehensive compilation of PIGE cross-section data for the most important elements used in IBA applications. About 500 datasets (new and existing data) have 
already been uploaded in IBANDL as can be seen in Fig. 2 and the updating procedure is still ongoing. Detailed assessments of the new data as well as of those existing in the literature will be included in the final technical report of the CRP.

Several outstanding issues have emerged during the course of the CRP that require further attention [7]:

- There is a need for measurements, in particular of angular distributions to support the evaluation procedure and produce recommended cross sections in cases where discrepancies are still not resolved.

- Evaluation efforts should be extended to include uncertainties in the evaluated files.

- Benchmarking experiments are needed to validate the measured and evaluated data.

- PIGE analysis codes should be incorporated in existing widely used IBA analysis codes.

In response to the above-mentioned data needs, the NDS held a Technical Meeting on Benchmarking Experiments for Ion Beam Analysis [22] from 26-29 May 2015. Participants gathered to discuss the validation of nuclear cross-section data for IBA through carefully planned benchmark measurements. The meeting produced a well-defined methodology for performing benchmark experiments, detailed guidelines for benchmarking experiments for the three techniques that were discussed, namely EBS, NRA and PIGE, and corresponding lists of priority benchmarking experiments. Details of the meeting will be published in the summary report which is in preparation. Presentations at the meeting are available from [22].

\section{References}

[1] CRP on Development of a Reference Database for Ion Beam Analysis, 20052010. <https://www-nds.iaea.org/iba/publiclBA.html>.
[2] Ion Beam analysis Nuclear Data Library (IBANDL). <http://www-nds.iaea.org/ ibandl/>.

[3] SigmaCalc Online Calculator. <http://sigmacalc.iate.obninsk.ru/>

[4] D. Abriola, A.P. Jesus, in: IAEA Consultant's Meeting on Assessment of Nuclear Data Needs for Particle Induced Gamma Ray Emission (PIGE), Vienna, Austria, INDC(NDS)-0568, 2010. <http://www-nds.iaea.org/publications/indc/indcnds-0568/>.

[5] D. Abriola, A.P. Jesus, in: 1st RCM of CRP on Reference Database for ParticleInduced Gamma-ray Emission spectroscopy, Vienna, Austria, INDC(NDS)-0589, 2011. <http://www-nds.iaea.org/publications/indc/indc-nds-0589/>.

[6] D. Abriola, P. Dimitriou, A.P. Jesus, in: 2nd RCM of CRP on Reference Database for Particle-Induced Gamma-ray Emission spectroscopy, Vienna, Austria, INDC (NDS)-0625, 2012. <http://www-nds.iaea.org/publications/indc/indc-nds$0625 />$.

[7] P. Dimitriou, A.P. Jesus, in: 3rd RCM of CRP on Reference Database for ParticleInduced Gamma-ray Emission spectroscopy, Vienna, Austria, INDC(NDS)-0664 2014. <http://www-nds.iaea.org/publications/indc/indc-nds-0664/>.

[8] J. Raisanen, P. Tikkanen, Nucl. Instr. Methods A 723 (2013) 5-7.

[9] M. Chiari, B. Melon, L. Salvestrini, M. Fonseca, E. Alves, A. Jesus, Nucl. Instr. Methods B 332 (2014) 355-358.

[10] A. Lagoyannis, K. Preketes-Sigalas, M. Axiotis, V. Foteinou, S. Harissopulos, M. Kokkoris, P. Misaelides, V. Paneta, N. Patronis, Nucl. Instr. Methods B 342 (2015) 271-276.

[11] I. Zamboni, Z. Siketić, M. Jakšić, I. Bogdanović Radović, Nucl. Instr. Methods B 342 (2015) 266-270.

[12] L. Csedreki, I. Uzonyi, Z. Szikszai, G. Gyürky, G.Á. Szki, Á.Z. Kiss, Nucl. Instr. Methods B 328 (2014) 20-26.

[13] L. Csedreki, I. Uzonyi, Z. Szikszai, G. Gyürky, G.Á. Szíki, Á.Z. Kiss, Nucl. Instr. Methods B 328 (2014) 59-64.

[14] L. Csedreki, Z. Szikszai, G.Á. Szíki, I. Kocsis, Nucl. Instr. Methods B 342 (2015) 184-187.

[15] L. Csedreki, R. Huszánk, Nucl. Instr. Methods B 348 (2014) 165.

[16] A. Gurbich, Nucl. Instr. Methods B 331 (2014) 31-33.

[17] EXFOR database. <http://www-nds.iaea.org/exfor/>.

[18] C. Angulo et al., Nucl. Phys. A 656 (1999) 3-187.

19] P. Dimitriou (Ed.), Developments in the Ion Beam Analysis Nuclear Data Library (IBANDL), Vienna, Austria, INDC(NDS)-0660, 2014. <http://www-nds. iaea.org/publications/indc/indc-nds-0660/>.

[20] Emitted Radiation Yield Analysis code (ERYA). <http://www.itn.pt/sec/fis/rn/ uk_rn_erya.htm>.

[21] M. Fonseca, in: these proceedings.

[22] IAEA Technical Meeting on Benchmarking Experiments for Ion Beam Analysis, 26-29 May 2015, Vienna, Austria. <http://www-nds.iaea.org/index-meetingcrp/TM-IBA-2015/>. 